\title{
Les comédies pastorales de Rotrou et l'histoire de leur réception
}

Dans l'article qu'elle consacre aux images de Rotrou construites par l'historiographie du théâtre français de 1674 à 1750, E. Mortgat-Longuet fait état d'une sélection rigoureuse parmi les pièces qui constituent l'œuvre du dramaturge ${ }^{1}$. Les critiques privilégient, en effet, ses tragédies - et certaines d'entre elles plus particulièrement (Hercule mourant, Antigone, Cosroès et Venceslas) - et de ses comédies ils ne retiennent guère que celles qui sont imitées de Plaute (Les Sosies, Les Captifs et Les Ménechmes) $)^{2}$. Comme le montre E. Mortgat-Longuet, une telle vision s'explique par le prestige dont jouit traditionnellement le genre tragique et, a contrario, par le discrédit dont souffre la comédie prémoliéresque $^{3}$ - à l'exception des rares pièces placées sous le haut patronage de l'Antiquité ${ }^{4}$. La hiérarchisation, qui sous-tend ainsi le discours critique, affecte les auteurs eux-mêmes. En effet, nombreux sont les commentateurs qui jugent les tragédies de Rotrou à l'aune de celles de Corneille, considéré alors comme l'une des meilleures incarnations du « classicisme » qui distingue le « siècle de Louis XIV $»^{5}$.

L'ensemble de ces représentations continue de peser dans l'histoire littéraire telle qu'elle s'élabore au dix-neuvième siècle ${ }^{6}$ - à quelques nuances près, toutefois, comme en témoigne l'exemple de Rotrou. Ainsi, après avoir été presque totalement ignorées des critiques, celles que l'on appellera plus tard «comédies pastorales»-La Diane, La Célimène, Le Filandre, La Clorinde et La Florimonde, pièces auxquelles on a coutume d'adjoindre La Céliane, malgré le nom de tragi-comédie que porte l'édition originale - font désormais l'objet d'analyses qui, sans leur être toujours favorables, laissent néanmoins augurer de leur réhabilitation à venir. C'est précisément le parcours critique de ces pièces que nous nous proposons de retracer, depuis les appréciations sévères formulées par les historiens du théâtre au dix-huitième siècle jusqu'aux études les plus récentes, centrées sur la problématique des genres. Loin de vouloir ainsi dénoncer l'aveuglement de critiques qui n'auraient pas su saisir l'intérêt de pièces réhabilitées par la suite, nous cherchons simplement à comprendre les enjeux liés aux interprétations successives qui en ont été données.

\section{De la redécouverte à la réhabilitation}

\footnotetext{
${ }^{1}$ Emmanuelle Mortgat-Longuet, «Images de Rotrou dans l'historiographie du théâtre français (1674-1750) », p. 285-300 dans Le Théâtre de Rotrou, actes du colloque de Tours (15-17 juin 2005), dir. Pierre Pasquier, Littératures classiques, 2007.

${ }^{2}$ Ibid., p. 296-297.

${ }^{3}$ Ibid., p. 295.

${ }^{4}$ Ibid., p. 297.

${ }^{5}$ Selon le titre que Voltaire donne à l'un de ses ouvrages, publié en 1740.

${ }^{6}$ Voir, à ce sujet, notre article «L'exemple d'auteurs 'préclassiques' redécouverts en France à la fin du XIX siècle : enjeux esthétiques et idéologiques », p. 111-125 dans L'Histoire littéraire au seuil du XXI siècle: controverses et consensus, dir. Luc Fraisse, Paris, P.U.F., 2005.
} 
Malgré leur parcimonie, les remarques relatives aux comédies pastorales de Rotrou que l'on rencontre dans les histoires du théâtre français publiées au dix-huitième siècle méritent toute notre attention. Dans ses Recherches sur les théâtres français, parues en 1735, Beauchamps dresse ainsi la liste des pièces du dramaturge, accompagnée de commentaires plus ou moins fournis, et, à propos de celles qui composent notre corpus, il use presque systématiquement de termes dépréciatifs : La Diane, dit-il, est « irrégulière », La Célimène " régulière », mais « froide et languissante », Le Filandre « froid» et La Clorinde «trop courte et froide » ${ }^{7}$. À la chaleur de la passion qui, conformément à la tradition du genre, se dégage des tragédies de Rotrou s'opposerait donc la froideur de pièces qui laisseraient ainsi le lecteur (ou le spectateur) insensible - un critère auquel s'ajoute celui de l'irrégularité, qui place La Diane, en l'occurrence, à l'écart du modèle classique. Dans leur Histoire du théâtre français, publiée en 1745, les frères Parfaict sont à peine plus indulgents à l'égard de ces pièces. Ils reprochent, en effet, à $L a$ Clorinde une intrigue à la fois « ridicule » et « très embrouillée $»^{8}$, et au Filandre les « ruses communes, et usées que [les] Amants emploient pour arriver à leurs fins, et qui ne servent cependant qu'à allonger l'intrigue du Poème $»^{9}$. Se trouvent ainsi dénoncés la confusion et le faible intérêt dramatique qui s'attachent à certains épisodes, en contradiction avec l'idéal de clarté et de rigueur qu'incarne le classicisme. Une opinion que vient confirmer le jugement sans appel prononcé au sujet de La Florimonde: une pièce, estiment les frères Parfaict, qui «ne fait guère honneur à Rotrou ; tant par le sujet, la conduite que la versification $»^{10}$.

De même nature sont les arguments que formule Viollet-le-Duc dans l'édition qu'il donne du Théâtre de Rotrou en $1820^{11}$. Dans les notices qui précèdent les pièces, le critique dénonce à son tour la faiblesse et la confusion des intrigues, notamment celles du Filandre ${ }^{12}$ et de La Diane ${ }^{13}$, et, en ce qui concerne La Clorinde, il attribue la peinture de «sentiments faux et quintessenciés » à l'inspiration romanesque de la comédie ${ }^{14}$. Toutefois, à la différence de ses prédécesseurs, il reconnaît à ces pièces des qualités qui touchent à la forme ou au contenu : il relève ainsi dans La Clorinde " plusieurs scènes d'amour ou de jalousie d'un excellent ton de comédie »" ${ }^{15}$ et dans La Célimène des concetti «fort spirituellement exprimés », qui illustrent les « progrès » du poète dans « l'art d'écrire $»^{16}$.

Précisément, Viollet-le-Duc développe de la chronologie des pièces de Rotrou une vision particulière, puisqu'il estime que l'ordre dans lequel elles furent publiées correspond très exactement à

\footnotetext{
${ }^{7}$ Pierre-François Godard de Beauchamps, Recherches sur les théâtres de France, Paris, Prault, 1735, p. 123-124.

${ }^{8}$ Claude et François Parfaict, Histoire du théâtre français, $1^{\text {re }}$ éd. 1734-1649, Genève, Slatkine Reprints, 1967, t. 1, vol. 5, p. 166 .

${ }^{9}$ Histoire du théâtre français, depuis son origine jusqu'à présent, t. 5, Paris, Le Mercier et Saillant, 1745, p. 114.

${ }^{10}$ Claude et François Parfaict, Histoire du théâtre français, op. cit., p. 252.

${ }^{11}$ Cuvres complètes de Jean Rotrou, t. 5, éd. d'Emmanuel Viollet-le-Duc, Paris, Desoer, 1820, Genève, Slatkine Reprints, 1967.

${ }^{12}$ Il y relève une « foule d'incidents ridicules » (ibid., t. 2, p. 527).

${ }^{13}$ Il la juge « presque inintelligible à la lecture » (ibid., t. 3, p. 265).

${ }^{14}$ Ibid., p. 173.

${ }^{15}$ Ibid., p. 172.

${ }^{16}$ Ibid., t. 2, p. 78.
} 
celui dans lequel elles furent créées ${ }^{17}$. Si cette coïncidence se vérifie en ce qui concerne les deux premières pièces, L'Hypocondriaque et La Bague de l'oubli ${ }^{18}$, et si l'ordre de composition a pu, jusqu'à un certain point, être respecté pour la publication des pièces suivantes, les incertitudes néanmoins demeurent nombreuses ${ }^{19}$. La chronologie est d'autant plus complexe que, comme l'a montré A. Howe à partir de l'examen des actes notariés relatifs à la carrière de Rotrou, la distance temporelle qui sépare la création de la publication est très variable suivant les pièces ${ }^{20}$. D'une mauvaise compréhension de la chronologie naissent des erreurs d'interprétation, qui affectent certaines des comédies pastorales de Rotrou, La Célimène en particulier, qui succéderait à des pièces inspirées de l'Antiquité (Les Ménechmes et Hercule mourant, publiés en 1636, quelques mois seulement avant elle), alors qu'elle leur est probablement antérieure ${ }^{21}$, et plus encore La Florimonde, qui, selon toute vraisemblance, est contemporaine de la précédente ${ }^{22}$, mais dont la publication tardive (en 1655) donne le sentiment qu'elle fait suite à la tragédie de Cosroès et à la tragi-comédie de Dom Lope de Cardone, publiées respectivement en 1649 et $1652^{23}$. À propos de ce «dernier ouvrage »-puisque telle est la formule par laquelle s'ouvre l'édition originale de La Florimonde ${ }^{24}$ - Viollet-le-Duc décrit ainsi le déclin d'un dramaturge à l'issue d'une période qu'il aurait pour l'essentiel consacrée à la tragédie et à la tragi-comédie, et au cours de laquelle son génie se serait manifesté avec éclat. Encore une fois, une telle lecture ne saurait être comprise hors de la hiérarchie traditionnelle des genres dramatiques, qui situe la tragédie au rang le plus haut et la comédie au rang le plus bas, mais elle participe également de l'idée, chère aux historiens de la littérature du dix-neuvième siècle, selon laquelle la succession des œuvres - pour un auteur, un genre ou une période donnée - doit suivre une courbe ascendante. Pour le reste, l'analyse conduite par le critique véhicule un certain nombre des préjugés traditionnellement attachés au genre romanesque, auquel on reproche tout à la fois ses artifices et son irrégularité - par opposition à la tragédie, en particulier, qu'il est possible de réduire au modèle de la tragédie régulière, et à laquelle on reconnaît volontiers une vérité dans la peinture des sentiments.

\footnotetext{
${ }^{17}$ Dans sa Bibliothèque des théâtres (Paris, Chardon et Briasson, 1733, p. 142), Maupoint déjà superposait les deux séries de dates.

${ }^{18}$ Voir, à ce sujet, l'introduction de Hélène Baby à L'Hypocondriaque, p. 19 dans Théâtre complet, t. 5, Paris, S.T.F.M., 2002; et l'introduction de Noémie Courtès à La Bague de l'oubli, p. 15-16 dans Théâtre complet, éd. cit., t. 9, 2007.

${ }^{19}$ Voir, à ce sujet, l'article de Perry Gethner, « La chronologie du théâtre de Rotrou. Mise au point », p. 242-257 dans Revue d'histoire du théâtre III, 1991, n 171.

${ }^{20}$ Écrivains de théâtre 1600-1649, documents réunis et présentés par Alan Howe, Paris, Centre historique des Archives nationales, 2005.

${ }^{21}$ Les achevés d'imprimer portent respectivement les dates du 15 mai, du 28 mai et du 8 octobre 1636. Selon les spécialistes, La Célimène a pu être créée entre 1630 et 1632 (voir, à ce sujet, l'introduction de Véronique Lochert, p. 10-11 dans Théâtre complet, éd. cit., t. 6, 2003), Les Ménechmes avant la fin de l'année 1634 (voir, à ce sujet, l'introduction de Jean-Yves Vialleton, p. 17 dans Théâtre complet, éd. cit., t. 8, 2005) et Hercule mourant en février 1634 (voir, à ce sujet, l'introduction de Dominique Moncond'huy, p. 17-18 dans Théâtre complet, éd. cit., t. 2, 1999).

${ }^{22}$ Voir, à ce sujet, notre introduction à la pièce (Théâtre complet, éd. cit., t. 10, 2010, p. 428-432).

${ }^{23}$ Viollet-le-Duc corrige ensuite l'affirmation initiale en déclarant à propos de La Florimonde : «L'époque de sa représentation paraît cependant être antérieure à celle de Don Lope de Cardone » (op. cit., t. 5, p. 411).

${ }^{24}$ La Florimonde, Paris, Sommaville, 1655.
} 
Malgré les nuances qu'elles apportent, les remarques que formulent Jarry et Curnier à propos des comédies pastorales de Rotrou dans les ouvrages qu'ils consacrent respectivement au dramaturge en 1868 et $1885^{25}$ ne se distinguent guère des précédentes, à tel point que certaines d'entre elles semblent avoir tout simplement été calquées sur celles de Viollet-le-Duc, telle cette phrase écrite par Curnier au sujet de La Clorinde: «Au milieu de sentiments faux et d'expressions alambiquées, on remarque quelques bonnes scènes de jalousie $»^{26}$. De même, le critique déplore la faiblesse de l'intrigue sur laquelle repose Le Filandre $e^{27}$, et l'impression de confusion qui se dégage de La Diane ${ }^{28}$ et de La Célimène ${ }^{29}$. À l'exemple de Jarry ${ }^{30}$, il regrette, par ailleurs, pour l'ensemble de ces pièces, des «développements poétiques", qui ne sont pas toujours "en harmonie avec le ton général de l'œuvre $\aleph^{31}$, et reproche au style de Rotrou une préciosité qui, à ses yeux, nuit à la qualité de la langue ${ }^{32}$. Si Jarry lui-même se déclare lassé par la « galanterie continuelle $»^{33}$ qui affecte le discours et le comportement des personnages, il apprécie néanmoins le charme que possèdent ces « œuvres de fantaisie» et l'élégance avec laquelle les amoureux s'y comportent ${ }^{34}$. Enfin, et surtout, le caractère faiblement comique de ces pièces empêche, selon les critiques, de reconnaitre à Rotrou la « verve comique $»^{35}$ dont il avait fait la brillante démonstration dans sa comédie à l'italienne de $\mathrm{La} S \propto e u r^{36}$ et qui, si elle s'était manifestée uniformément dans son œuvre comique, aurait permis de le considérer comme un véritable auteur de comédie, au même titre que Molière par exemple.

De manière significative, les rares mérites que Curnier attribue aux comédies pastorales de Rotrou le sont uniquement en référence aux pièces sérieuses dont le dramaturge est également l'auteur. Il remarque ainsi dans une pièce «médiocre » comme La Clorinde de "nobles élans » analogues à ceux que l'on perçoit avec plus d'évidence dans Venceslas ${ }^{37}$. À l'instar de Viollet-le-Duc, Jarry et Curnier établissent donc une véritable hiérarchie entre des pièces-les tragédies principalement - qu'ils jugent presque à l'égal de celles de Corneille ${ }^{38}$ et des pièces qu'ils considèrent, sinon comme de malheureux accidents, du moins comme de simples parenthèses dans la carrière d'un dramaturge dont la tragédie aurait été sans conteste le genre de prédilection ${ }^{39}$.

\footnotetext{
25 Jules Jarry, Essai sur les ceuvres dramatiques de Jean Rotrou, Lille, Quarré, Paris, Durand, [s.d.] ; Léonce Curnier, Étude sur Jean Rotrou, Paris, typogr. A. Hennuyer, 1885.

${ }^{26}$ Ibid., p. 194.

${ }^{27}$ Ibid., p. 189.

${ }^{28}$ Ibid., p. 143-145.

${ }^{29}$ Ibid., p. 161-162.

${ }^{30}$ Jules Jarry, op. cit., p. 66 et 275.

${ }^{31}$ Léonce Curnier, op. cit., p. 196. C'est ainsi qu'il qualifie La Clorinde d'« idylle » (ibid.).

32 Sans parler de "préciosité », Curnier évoque la "phraséologie sentimentale qui était en honneur dans un certain monde » (ibid., p. 33). Jarry, quant à lui, décrit ironiquement le « beau langage des amants » (op. cit., p. 63).

${ }^{33}$ Ibid.

${ }^{34}$ Ibid., p. 60-63.

${ }^{35}$ Léonce Curnier, op. cit., p. 192.

${ }^{36}$ Ibid.

${ }^{37}$ Ibid., p. 119.

${ }^{38}$ Voir, à ce sujet, notre article précédemment cité.

${ }^{39}$ Jules Jarry, op. cit., p. 210 et 259-260.
} 
Sans être toujours explicite, la référence en réalité est constante à la tragédie et au classicisme auquel ce genre est communément associé. L'efficacité qui caractérise la dramaturgie classique, la simplicité de la langue que l'on reconnaît volontiers aux œuvres tragiques expliquent, en effet, pour une large part les critiques adressées aux comédies pastorales de Rotrou que nous venons de rapporter. La mixité générique, qui distingue ces pièces, va également à l'encontre du principe de séparation des genres, hérité du classicisme, et représente de surcrô̂t un obstacle majeur au regard d'un lecteur qui entreprendrait d'identifier très précisément la catégorie à laquelle elles appartiennent. Enfin, l'humour et la « légèreté ${ }^{40}$ qui s'en dégagent heurtent l'image d'auteur sérieux que les historiens et biographes se sont évertués à construire autour de la figure de Rotrou et que les récits d'une mort qualifiée d'hérö̈que ont sans aucun doute contribué à entretenir ${ }^{41}$. Au vu de tous ces critères, les comédies pastorales de Rotrou ne sauraient donc être placées sur le même plan que les pièces sérieuses qui lui sont dues, en raison même de leur appartenance au genre de la comédie, mais, d'un autre côté, elles ne sauraient être considérées comme un exemple de réalisation dans le domaine de la comédie, étant donné la part réduite qu'elles accordent au comique, généralement retenu pour être un des traits distinctifs de ce genre. Soucieux de donner à Rotrou la place qu'il mérite dans l'histoire du théâtre français, Viollet-le-Duc et ses successeurs immédiats préfèrent ainsi mettre l'accent sur des pièces propres à donner l'image, valorisante, d'une œuvre à la fois cohérente et fondée sur le genre le plus prestigieux qui soit.

Dans l'histoire de la réception des comédies pastorales de Rotrou, un premier tournant se situe en 1905, avec la parution de l'ouvrage que Marsan consacre à la pastorale dramatique française des seizième et dix-septième siècles ${ }^{42}$, et qui participe de la volonté, commune à de nombreux critiques de sa génération, de réhabiliter des formes littéraires jusqu'alors dépréciées voire ignorées. Marsan met ainsi en lumière la coloration pastorale qui caractérise les pièces de Rotrou, mais surtout il les compare aux comédies urbaines de Corneille, elles-mêmes issues du modèle pastoral, sans pour autant nier la prédilection du premier pour le romanesque ${ }^{43}$. Il semble donc que la référence à Corneille soit nécessaire à la réhabilitation du théâtre de Rotrou, y compris dans le genre de la comédie, auquel le « grand » auteur est rarement associé ${ }^{44}$. L'intérêt qui se manifeste ainsi pour la pastorale se confirmera par la suite ${ }^{45}$, et trouve désormais un prolongement dans les études consacrées à l'esthétique galante, qui, dans les œuvres d'inspiration pastorale, détermine le langage et le comportement des personnages $^{46}$, et à laquelle les pièces de Rotrou elles-mêmes ne sont pas étrangères.

\footnotetext{
${ }^{40}$ Ibid., p. 61, 63 et 208.

${ }^{41}$ Il s'agit là d'un lieu commun, dont se font l'écho de nombreux critiques.

${ }^{42}$ Jules Marsan, La Pastorale dramatique en France à la fin du XVI et au commencement du XVII siècle, Paris, Hachette, 1905.

${ }^{43}$ Ibid., p. 365.

${ }^{44}$ Le parallèle est repris par Robert Garapon, «Rotrou et Corneille », p. 385-394 dans Revue d'Histoire Littéraire de la France, octobre-décembre 1950.

${ }^{45}$ Parmi les travaux les plus récents figurent ceux de Daniela Dalla Valle, de Stéphane Macé et de JeanPierre Van Elslande.

${ }^{46}$ Voir, en particulier, l'ouvrage d'Alain Viala, La France galante : essai historique sur une catégorie culturelle, des origines jusqu'à la Révolution, Paris, P.U.F., 2008.
} 


\section{De l'introduction de la catégorie du baroque à la poétique du genre}

C'est évidemment avec l'introduction de la catégorie du baroque dans les études littéraires que les comédies pastorales de Rotrou ont enfin été reconnues à leur juste valeur. Dès lors, l'impression de confusion, que leur lecture faisait naître, est perçue comme le signe d'une complexité qui permet de voir en elles l'expression de l'irrégularité propre à l'esthétique baroque. C'est dans cette perspective que s'inscrit l'étude que J.-C. Vuillemin consacre à l'œuvre de Rotrou en 1994, Baroquisme et théâtralité, même si l'analyse que lui inspirent les comédies pastorales porte essentiellement sur la peinture du sentiment amoureux ${ }^{47}$.

La voie avait été ouverte par J. Morel dans son ouvrage Jean Rotrou, dramaturge de l'ambiguïté, publié en $1968^{48}$, mais à la référence au baroque s'y ajoute une méthode, issue du structuralisme, qui rend le critique particulièrement attentif à la forme des textes. Ce faisant, il vise à dégager l'unité organique de l'œuvre, conçue comme un ensemble relativement homogène malgré l'évidente disparité des éléments qui le constituent. Il s'attache ainsi à mettre en lumière un certain nombre de motifs, comme celui de l'amour, qu'avait déjà étudié W. Leiner à propos de Rotrou ${ }^{49}$ et dont les déclinaisons (le romanesque et le réalisme) ne sont pas nécessairement liées à l'appartenance générique des pièces ${ }^{50}$. La complexité qui distingue la structure des comédies pastorales de Rotrou se trouve, en outre, réduite au profit d'un schéma dramatique unique, fondé sur le passage du déséquilibre à l'équilibre (ou du désordre à l'ordre) ${ }^{51}$, perceptible malgré la richesse et la variété des ornements que l'auteur se plaît à y insérer. Plus encore, le principe de la « conquête de la vérité à travers une feinte $\aleph^{52}$, qui détermine dans ces pièces l'enchaînement des faits, se rencontre dans l'ensemble de l'œuvre du dramaturge, comme par exemple dans sa tragédie du Véritable Saint Genest $t^{3}$. Toutefois, à l'image de ses prédécesseurs, J. Morel rapproche les pièces de Rotrou de celles de Corneille ${ }^{54}$, comme pour mieux montrer l'importance du rôle que, conjointement, elles ont joué dans la constitution d'un nouveau type de comédie, préférant le plaisant au comique.

C. Scherer, dans l'ouvrage qu'elle consacre à la comédie sous Louis XIII, reprend le parallèle, mais, à la «dramaturgie du quotidien », qu'incarnent les comédies urbaines de Corneille, elle oppose la « dramaturgie de l'imaginaire », que représentent les comédies pastorales de Rotrou ${ }^{55}$. Encore une

\footnotetext{
${ }^{47}$ Jean-Claude Vuillemin, Baroquisme et théâtralité : le théâtre de Jean Rotrou, Tübingen, Narr (Biblio 17), 1994.

${ }^{48}$ Jacques Morel, Jean Rotrou dramaturge de l'ambiguïté, Paris, Colin, 1968.

${ }^{49}$ Wolfang Leiner, «Deux aspects de l'amour dans le théâtre de Jean Rotrou : le romanesque et le réalisme », p. 179-204 dans Revue d'Histoire du Théâtre 11, 1959.

${ }^{50}$ Jacques Morel, op. cit., p. 30 et sqq.

${ }^{51}$ Ibid., p. 150 et 168 .

${ }^{52}$ Ibid., p. 168.

${ }^{53}$ Ibid., p. 58.

${ }^{54}$ Ibid., p. 137.

${ }^{55}$ Colette Scherer, Comédie et société sous Louis XIII. Corneille, Rotrou et les autres, Paris, Nizet, 1983, p. 55, 181, etc. Elle compare La Diane et La Suivante (ibid., p. 139-141).
} 
fois, il semble donc que la référence à Corneille soit inévitable, alors même que, comme le souligne C. Scherer, les différences sont grandes entre les deux séries de pièces.

Les travaux actuels dédiés aux comédies pastorales de Rotrou se situent dans le prolongement de l'étude qu'avait menée à leur sujet J. Morel. L'édition du Théâtre complet, dirigée par G. Forestier ${ }^{56}$, répartit ainsi les pièces suivant la typologie jadis établie par le critique ${ }^{57}-$ avec une place toute particulière accordée à La Clorinde, que la présence du soldat fanfaron empêche de considérer comme une «pastorale pure », selon le mot de $\mathrm{J}$. Morel ${ }^{58}$, et qui, de ce fait, se trouve associée à La Bague de l'oubli et à La Belle Alphrède, alors même que ces deux autres pièces correspondent à des conceptions de la comédie radicalement différentes ${ }^{59}$. La conduite de ce projet exprime également la vision totalisante d'une œuvre conçue comme un tout en dépit de la diversité des genres dans lesquels le dramaturge s'est tour à tour illustré et de la variété des formes prises par chacun d'entre eux. On retrouve ainsi, dans les commentaires eux-mêmes, des développements sur le thème du théâtre dans le théâtre, récurrent chez Rotrou, et une série de réflexions portant sur le statut générique de pièces pour lesquelles l'appartenance au genre de la comédie (ou de la tragi-comédie, en ce qui concerne $L a$ Céliane) est éminemment problématique ${ }^{60}$.

De manière générale, les modes de dénomination successifs choisis par les critiques pour désigner ces pièces varient en fonction de l'intérêt qu'elles suscitent, mais ils sont également le reflet des interprétations diverses dont elles ont pu faire l'objet depuis le dix-huitième siècle. En effet, si longtemps elles ont été appelées simplement comédies - conformément au terme qui figure dans les éditions originales (à l'exception de La Céliane), et dont il est d'ailleurs difficile de savoir au juste s'il est imputable à l'auteur, à l'éditeur et/ou à l'imprimeur ${ }^{61}-\mathrm{J}$. Morel et ses successeurs ont ensuite cherché des expressions susceptibles de rendre compte de leur spécificité (« pastorale parisienne», selon la formule inventée par L. Picciola à propos de La Diane $e^{62}$, «comédies de couleur pastorale », « comédies de type pastoral», et même «pastorales pures », suivant la terminologie de J. Morel ${ }^{63}$ ), quitte à briser la frontière qui, théoriquement, sépare les « comédies » proprement dites de la « tragicomédie » de La Céliane. Cette terminologie flottante témoigne, du reste, de la difficulté qu'il y a à définir de manière rigoureuse des pièces qui tendent à échapper aux catégories traditionnelles. Si, à présent, la volonté est de trouver des modes de dénomination qui se rapprochent le plus possible des appellations initiales, à l'inverse, on rencontre, sous la plume des premiers commentateurs, des qualificatifs qui s'en éloignent résolument et qui, de ce fait, sont chargés de signification. Maupoint,

\footnotetext{
${ }^{56}$ Théâtre complet, Paris, S.T.F.M., 1998-.

${ }^{57}$ La Diane et La Célimène sont ainsi réunies dans le tome 6 (2003) ; La Céliane, Le Filandre et La Florimonde le seront dans le tome 10 .

${ }^{58}$ Ibid., p. 137.

${ }^{59}$ Théâtre complet, éd. cit., t. 9, 2007.

${ }^{60}$ Voir, à ce sujet, l'analyse de Jean-Yves Vialleton dans son introduction à La Céliane, Théâtre complet, éd. cit., t. 10, p. 81-107.

${ }^{61}$ Voir, à ce sujet, notre article « La mixité des genres dramatiques dans le théâtre de Rotrou », p. 97-106 dans Le Théâtre de Rotrou, op. cit.

${ }^{62}$ Théâtre complet, éd. cit., t. 6, p. 213.

${ }^{63}$ Jacques Morel, op. cit., p. 109 et 168.
} 
dans sa Bibliothèque des théâtres, publiée en 1733, multiplie ainsi les lapsus révélateurs : il appelle $L a$ Céliane «comédie ", La Florimonde «tragi-comédie »-suggérant par là même une possible inversion des termes - et $L a$ Célimène « pastorale », en référence à l'Amarillis ${ }^{64}$, la pièce que Tristan rédigea à partir de la version initiale laissée par le futur auteur de la comédie ${ }^{65}$, et que l'on attribue parfois à ce dernier ${ }^{66}$.

Même si la tendance actuelle est donc de revaloriser des pièces longtemps dépréciées, les préjugés qui ont longtemps prévalu à leur sujet n'ont pas définitivement disparu, comme le montrent les pages que C. Mazouer leur consacre dans le premier volet de son Histoire du théâtre français au dix-septième siècle et qui se concluent par cette phrase : «Ce n'est décidément pas en cultivant cette pastorale que Rotrou pourrait séduire $\aleph^{67}$.

L'histoire de la réception des comédies pastorales de Rotrou depuis le dix-huitième siècle est riche d'enseignements, la rareté et la brièveté des premiers commentaires étant tout aussi significatives que les longues études qui leur ont été dédiées par la suite. À propos de ces pièces, s'est constitué un ensemble de lieux communs, qui leur fut longtemps préjudiciable. On reprocha, en effet, à leurs intrigues une confusion qui rendait leur lecture difficile, mais, surtout, les critiques qui leur furent adressées révèlent le mépris dont eurent à souffrir certaines formes littéraires, comme la pastorale et le roman, que la référence (explicite ou non) à L'Astrée permet d'associer étroitement. Si l'introduction de la catégorie du baroque a ensuite permis de montrer les mécanismes propres aux comédies pastorales de Rotrou, et si la faiblesse des intrigues a pu être mise sur le compte de cette dramaturgie de la gratuité ${ }^{68}$ qui caractérise une partie de son œuvre, de nombreuses questions restent encore en suspens, notamment sur le rapport entre la réalité des textes et le mode de dénomination qui leur a été choisi. Sans doute conviendrait-il aussi de considérer ces comédies pastorales pour elles-mêmes, indépendamment de Corneille, afin de montrer à quel point le théâtre de Rotrou excède les limites habituellement assignées aux genres dramatiques. La lecture de ces pièces invite, pour le moins, à jeter un regard nouveau sur l'œuvre du dramaturge dans son ensemble et à corriger un peu l'image d'auteur sérieux construite par l'histoire littéraire.

Sandrine Berrégard,

Université de Strasbourg

\footnotetext{
${ }^{64}$ La confusion était déjà dans Évrard Titon du Tillet, Description du Parnasse français, Paris, Coignard, 1727, p. 313.

${ }^{65}$ Voir, à ce sujet, Euvres complètes, t. 5, Paris, Champion (Sources classiques), 1999, p. 113 et 121.

${ }^{66}$ Maupoint, op. cit., p. 68 et 141.

${ }^{67}$ Charles Mazouer, Le Théâtre français de l'âge classique, t. 1 Le Premier XVII eiècle, Paris, Champion, Dictionnaires et Références, 2006, p. 503. Nous soulignons.

${ }^{68}$ Voir, à ce sujet, Jacques Morel, op. cit., p. 200-201.
} 
